\title{
Structure-Processing-Property Relationships of 3D Printed Porous Polymeric Materials
}

Ciera E. Cipriania ${ }^{a}$ Taekwang Ha ${ }^{b, c}$, Oliver B. Martinez Defillóa ${ }^{a}$, Manoj Mynenid, Yifei Wang ${ }^{a}$, Chandler C. Benjamin ${ }^{d}$, Jyhwen Wang ${ }^{\mathrm{d}, \mathrm{e}, *}$, Emily B. Pentzer ${ }^{\mathrm{a}, \mathrm{f,},}$, Peiran Wei ${ }^{\mathrm{a},{ }^{*}}$

aDepartment of Materials Science and Engineering, Texas A\&M University, College Station, TX, USA

bDepartment of Multidisciplinary Engineering, Texas A\&M University, College Station, TX, USA

'Department of Mechanical and Industrial Engineering, Norwegian University of Science and Technology, Trondheim, Norway

dDepartment of Mechanical Engineering, Texas A\&M University, College Station, TX, USA eDepartment of Engineering Technology and Industrial Distribution, Texas A\&M University, College Station, TX, USA

fDepartment of Chemistry, Texas A\&M University, College Station, TX, USA

*Correspondence: jwang@tamu.edu, emilypentzer@tamu.edu, peiran@tamu.edu

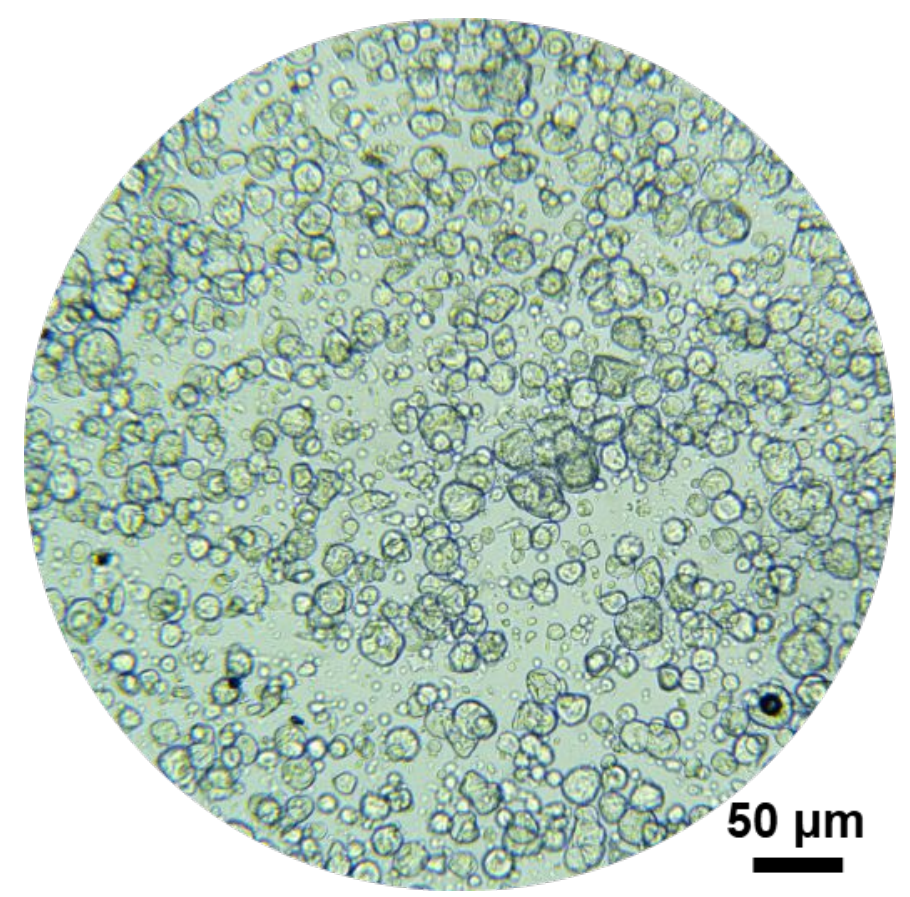

Figure S1. Optical microscopy image of paraffin wax beads in resin. 


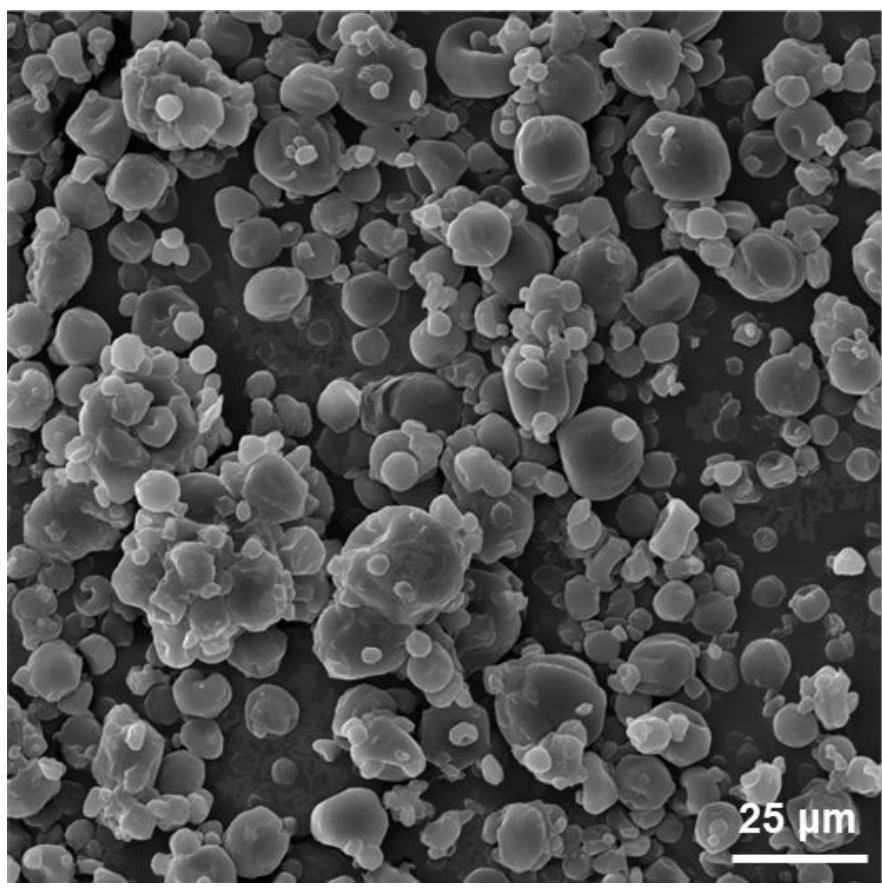

Figure S2. SEM image of paraffin beads.

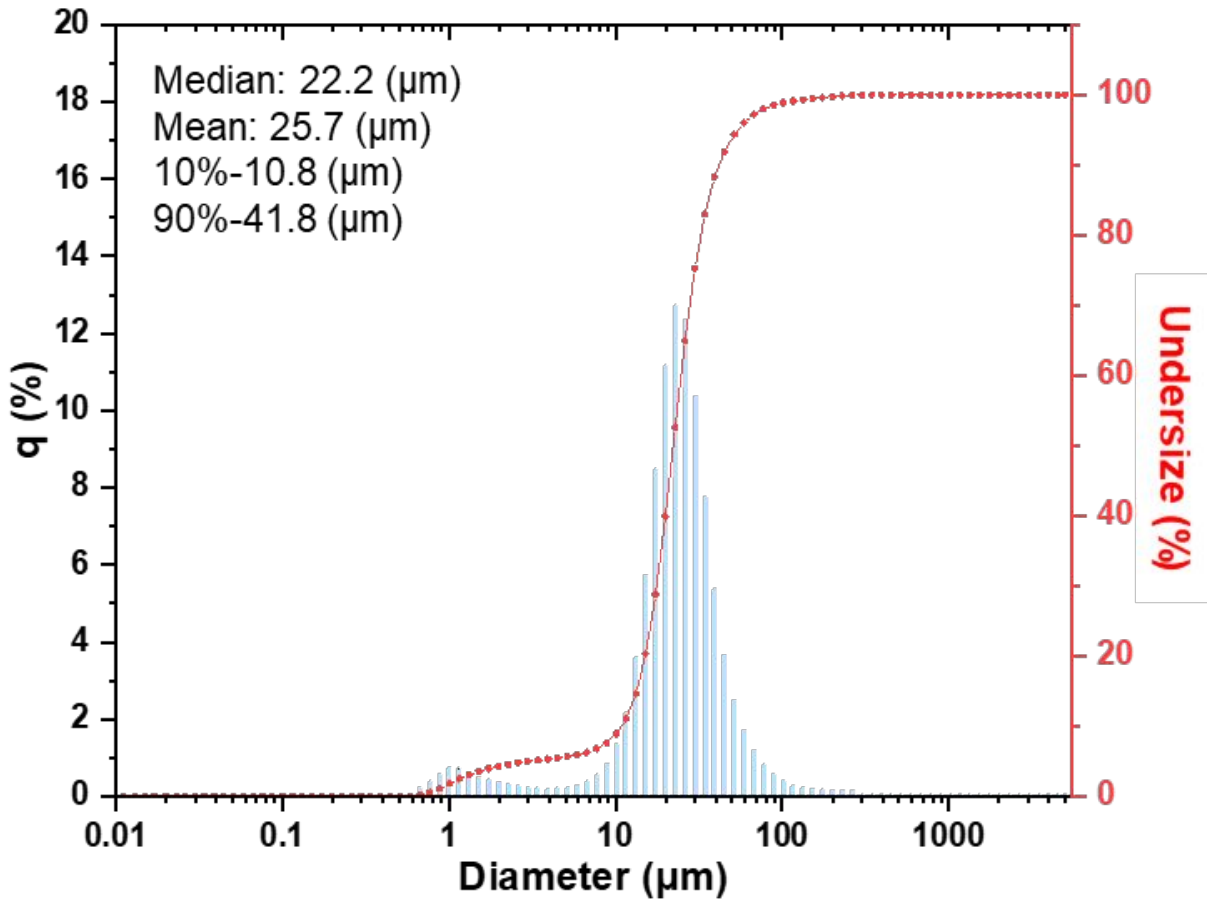

Figure S3. Size distribution histogram of the paraffin wax beads. 


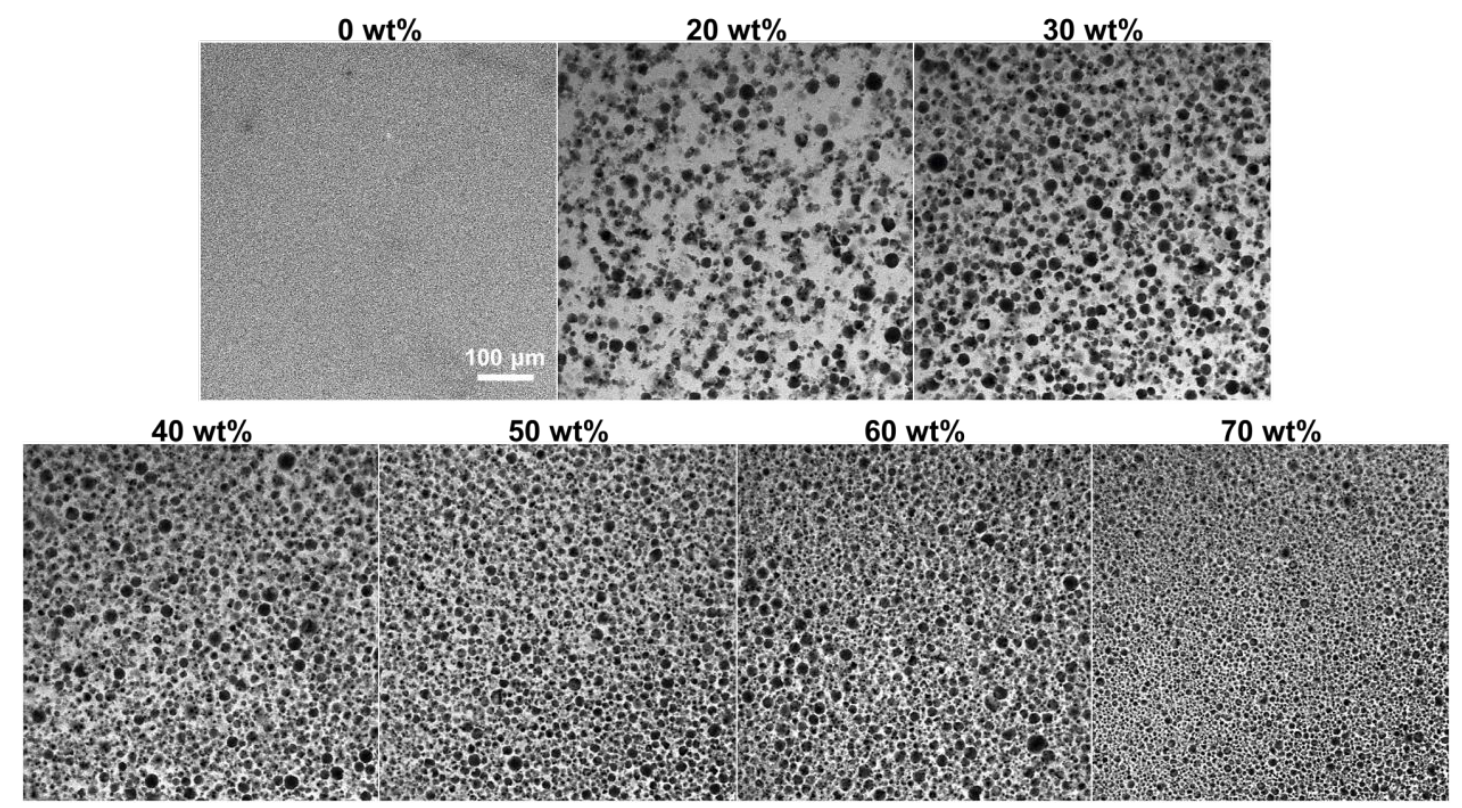

Figure S4. Confocal microscopy images of paraffin/photopolymer composite inks with varying $w t \%$ paraffin loading. 

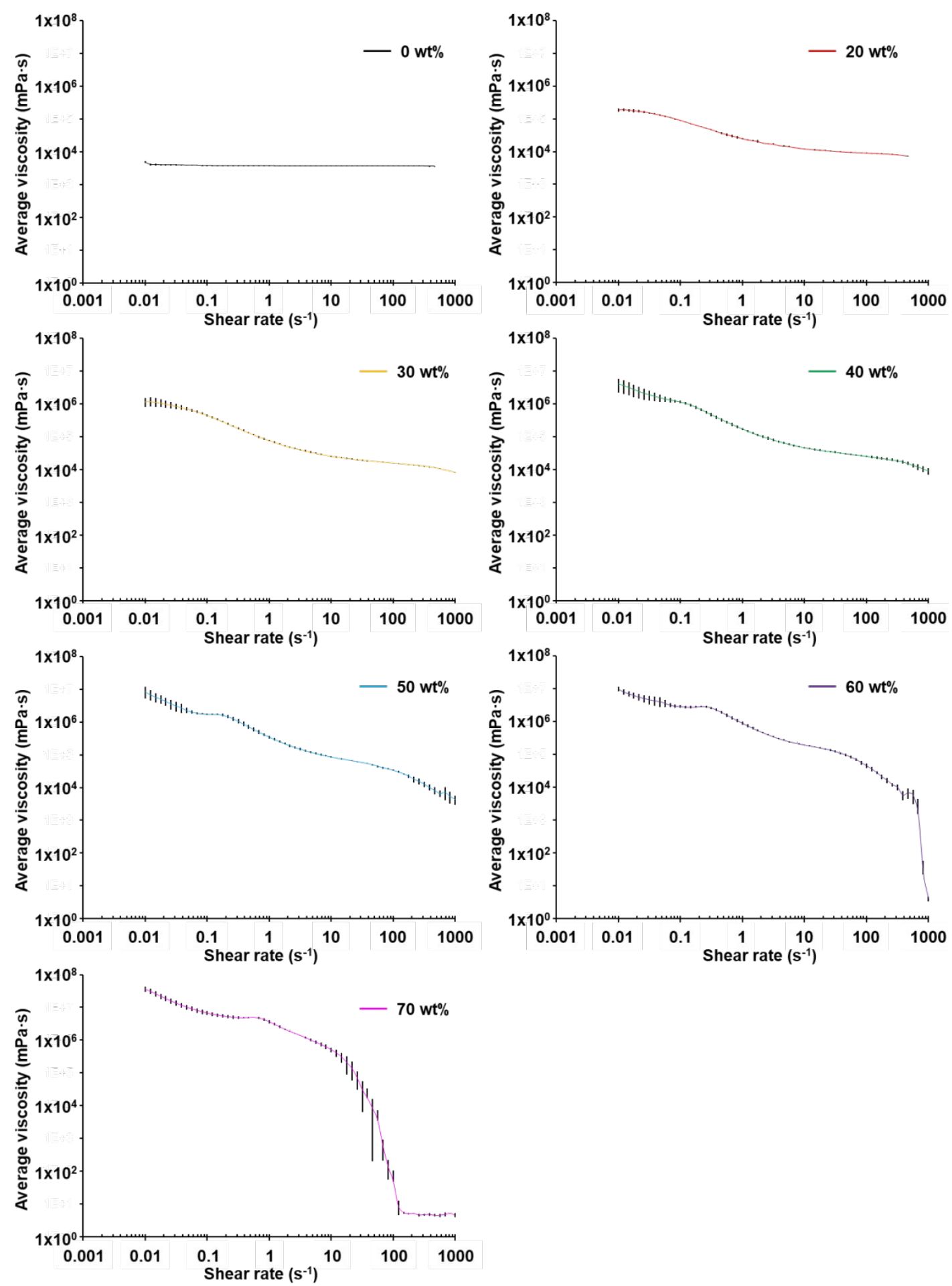

Figure S5. Average viscosity of each ink as a function of shear rate with error bars $(n=3)$. 


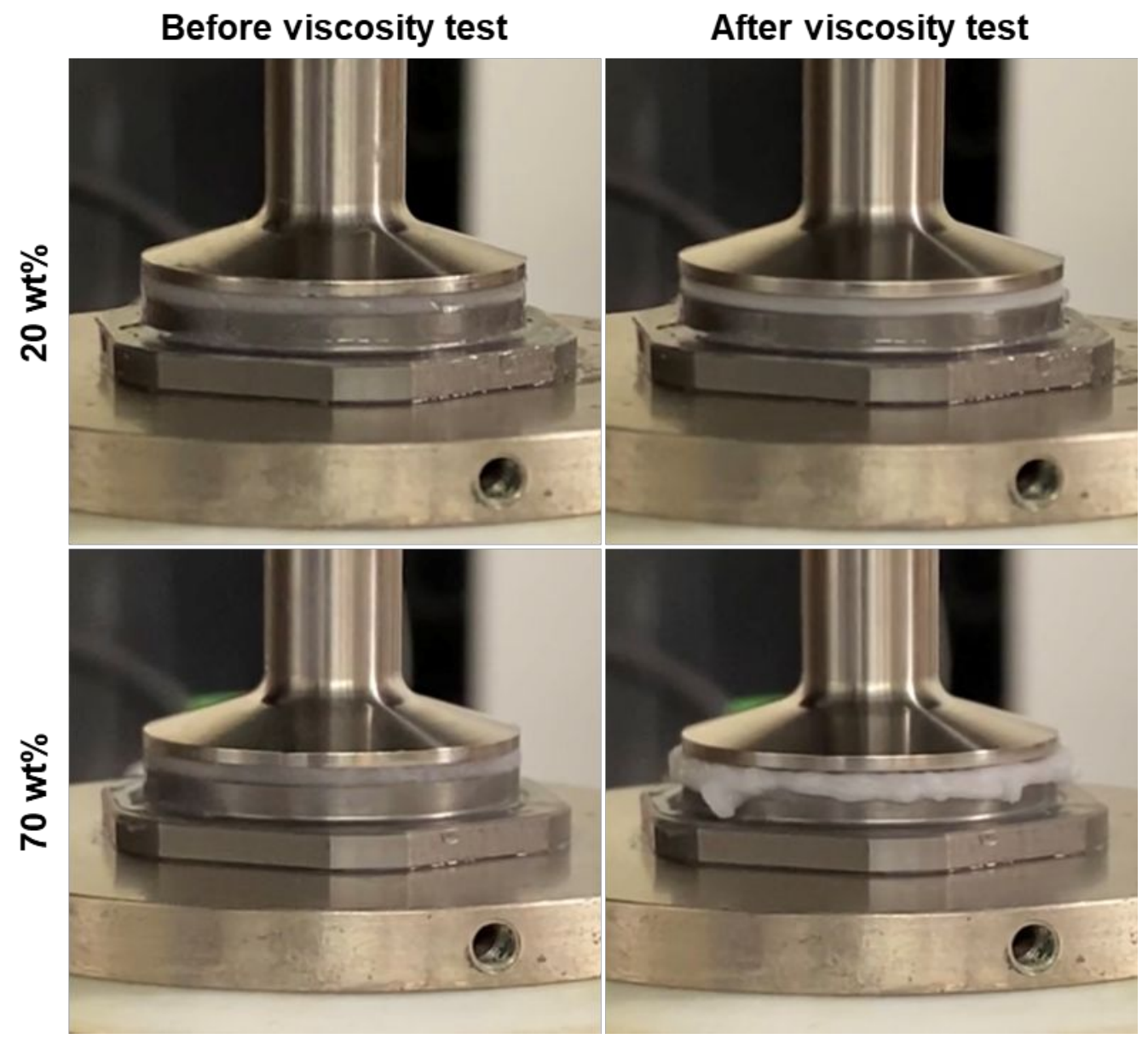

Figure S6. Digital images of inks containing 20 and $60 \mathrm{wt} \%$ paraffin before (left) and after (right) the viscosity-shear rate test. Upon being exposed to high shear rates, the ink with high paraffin loading was expelled from the sides of the parallel plates of the rheometer.

Table S1. The average depth of cure of each ink after exposure to UV light for 1 minute $(n=3)$. Percentage DOCs were calculated by setting the bulk resin DOC (0 wt\% paraffin loading) equal to $100.00 \%$ and subsequent samples relative to the bulk resin value.

\begin{tabular}{ccc}
\hline $\begin{array}{c}\text { Paraffin } \\
\text { loading } \\
(\mathrm{wt} \%)\end{array}$ & $\begin{array}{c}\text { Average DOC } \\
(\mathrm{mm})\end{array}$ & $\begin{array}{c}\text { Average DOC } \\
(\%)\end{array}$ \\
\hline 0 & $7.81 \pm 0.34$ & $100.00 \pm 4.37$ \\
20 & $6.83 \pm 0.32$ & $87.48 \pm 4.16$ \\
30 & $5.14 \pm 0.36$ & $65.74 \pm 4.63$ \\
40 & $3.95 \pm 0.66$ & $50.59 \pm 8.50$ \\
50 & $3.17 \pm 0.16$ & $40.56 \pm 2.06$
\end{tabular}



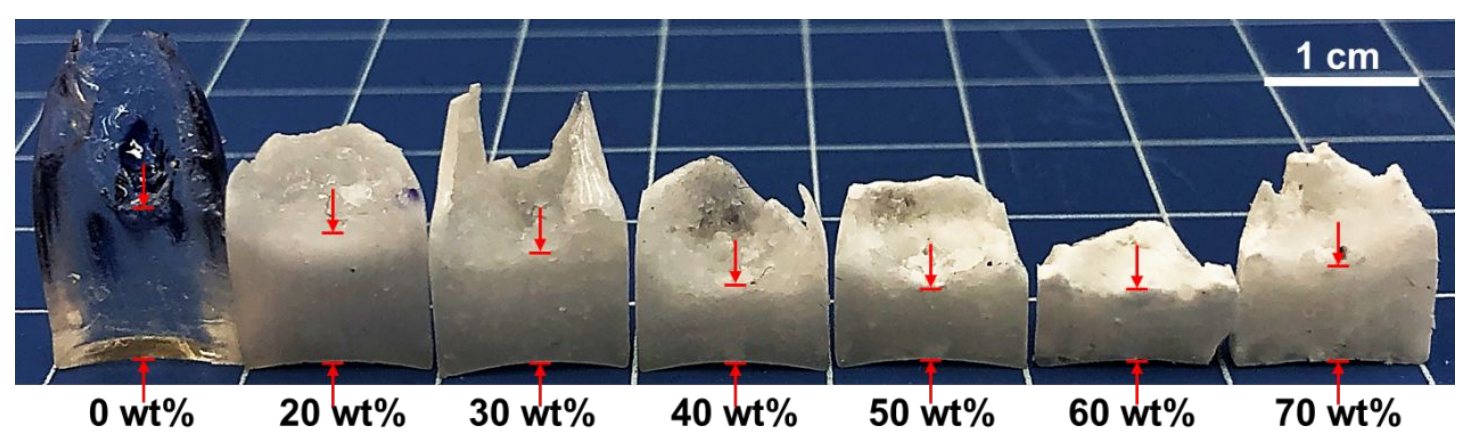

Figure S7. Digital photo of cured inks with different paraffin loading after 60 seconds of UV exposure and removal of uncured ink.

Table S2. Average DOCs of $60 \mathrm{wt} \%$ paraffin ink at different exposure times $(n=3)$.

\begin{tabular}{cc}
\hline $\begin{array}{c}\text { Time } \\
(\mathrm{s})\end{array}$ & $\begin{array}{c}\text { Average DOC } \\
(\mathrm{mm})\end{array}$ \\
\hline 0 & $0 \pm 0$ \\
30 & $2.17 \pm 0.17$ \\
60 & $3.01 \pm 0.19$ \\
90 & $4.54 \pm 0.52$ \\
120 & $6.23 \pm 0.38$ \\
150 & $6.70 \pm 0.15$ \\
180 & $6.84 \pm 0.05$ \\
\hline
\end{tabular}




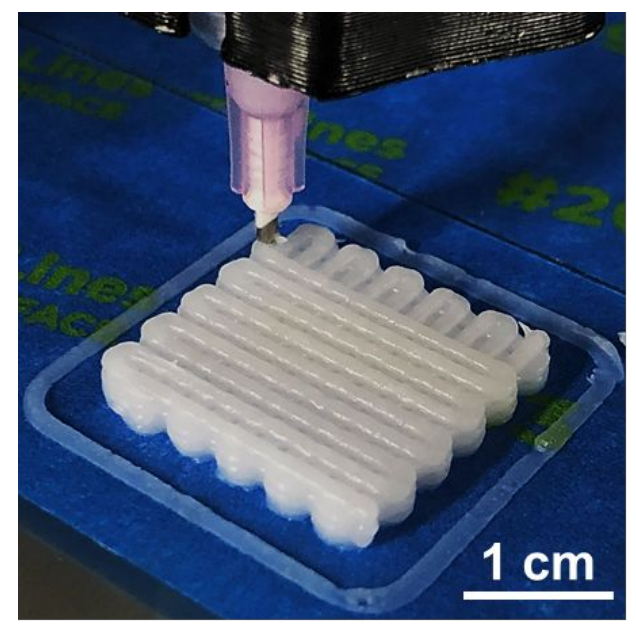

Figure S8. Digital image of a cubic lattice of $60 \mathrm{wt} \%$ ink being printed.

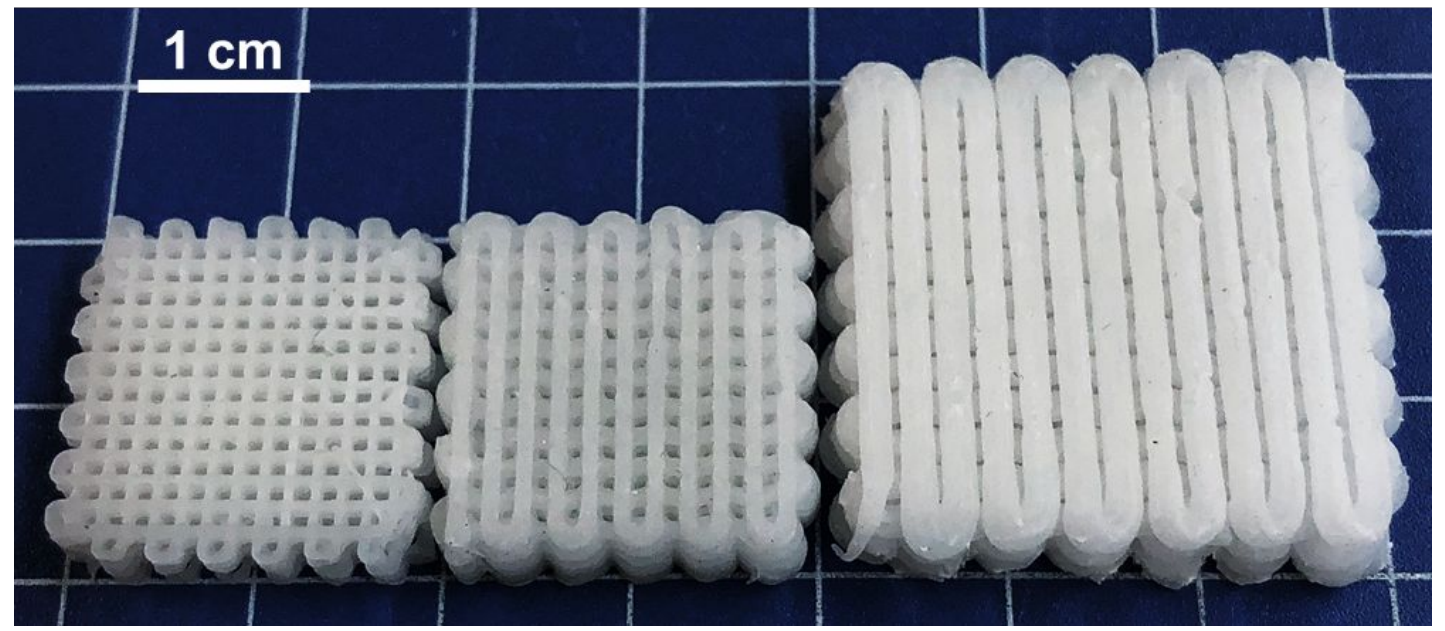

Figure S9. Digital image of 3D printed cured cubic lattices with different resolutions before filler removal. Left: $50 \mathrm{wt} \%$ ink printed with $22 \mathrm{G}$ nozzle; middle: $60 \mathrm{wt} \%$ ink printed with $18 \mathrm{G}$ nozzle; $70 \mathrm{wt} \%$ ink printed with $14 \mathrm{G}$ nozzle.

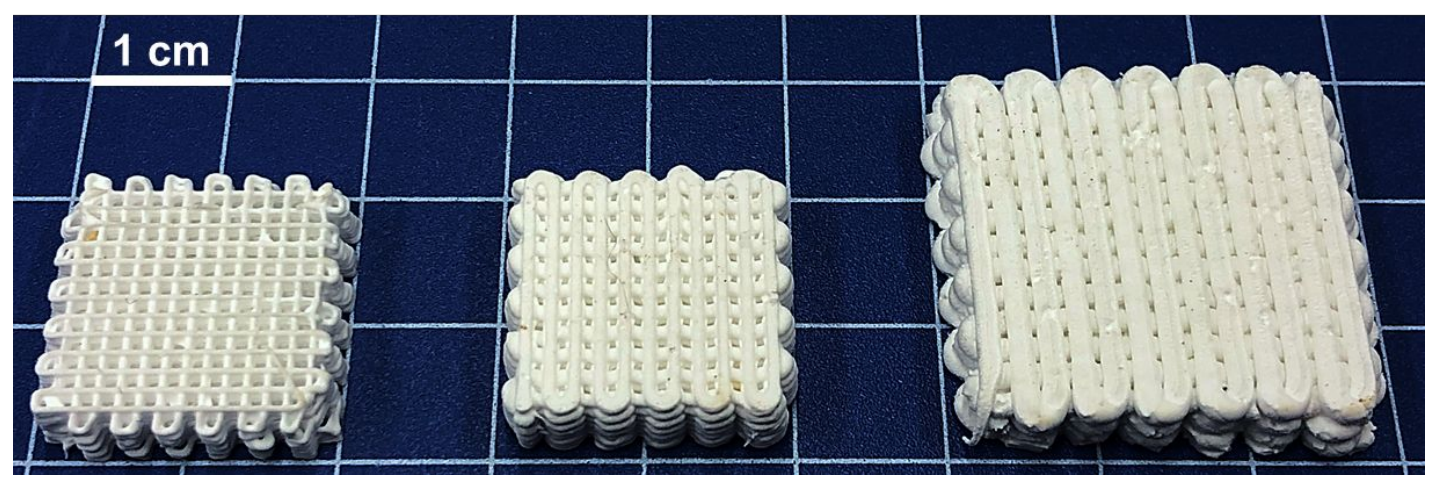

Figure S10. Digital photo of 3D printed cubic lattices with different resolutions after filler removal. Left: 53 vol\% porosity printed with $22 \mathrm{G}$ nozzle; middle: 63 vol\% porosity printed with $18 \mathrm{G}$ nozzle; 73 vol\% porosity printed with $14 \mathrm{G}$ nozzle.

Table S3. Weight of samples of $60 \mathrm{wt} \%$ paraffin ink before and after removal of paraffin, 
experimental porosity based on this weight loss, and theoretical porosity based on ink composition.

\begin{tabular}{lcccc}
\hline & $\begin{array}{c}\text { Weight before extraction } \\
(\mathrm{g})\end{array}$ & $\begin{array}{c}\text { Weight after extraction } \\
(\mathrm{g})\end{array}$ & $\begin{array}{c}\Delta_{\mathrm{wt}} \\
(\mathrm{g})\end{array}$ & $\begin{array}{c}\text { Experimental } \\
\text { porosity (vol\%) }\end{array}$ \\
\hline Sample 1 & 1.0828 & 0.4115 & 0.6713 & 65.0 \\
Sample 2 & 1.0594 & 0.4040 & 0.6554 & 64.8 \\
Sample 3 & 1.1004 & 0.4285 & 0.6719 & 64.1 \\
\hline Average & 1.0809 & 0.4147 & 0.6662 & 64.6 \\
Error & 0.0206 & 0.0126 & 0.0094 & 0.5 \\
Paraffin wax loaded in composite: $2.3990 \mathrm{~g}$ & & & \\
Resin loaded in composite: $1.5896 \mathrm{~g}$ & & & \\
Theoretical porosity: 63.2 vol\% & & &
\end{tabular}

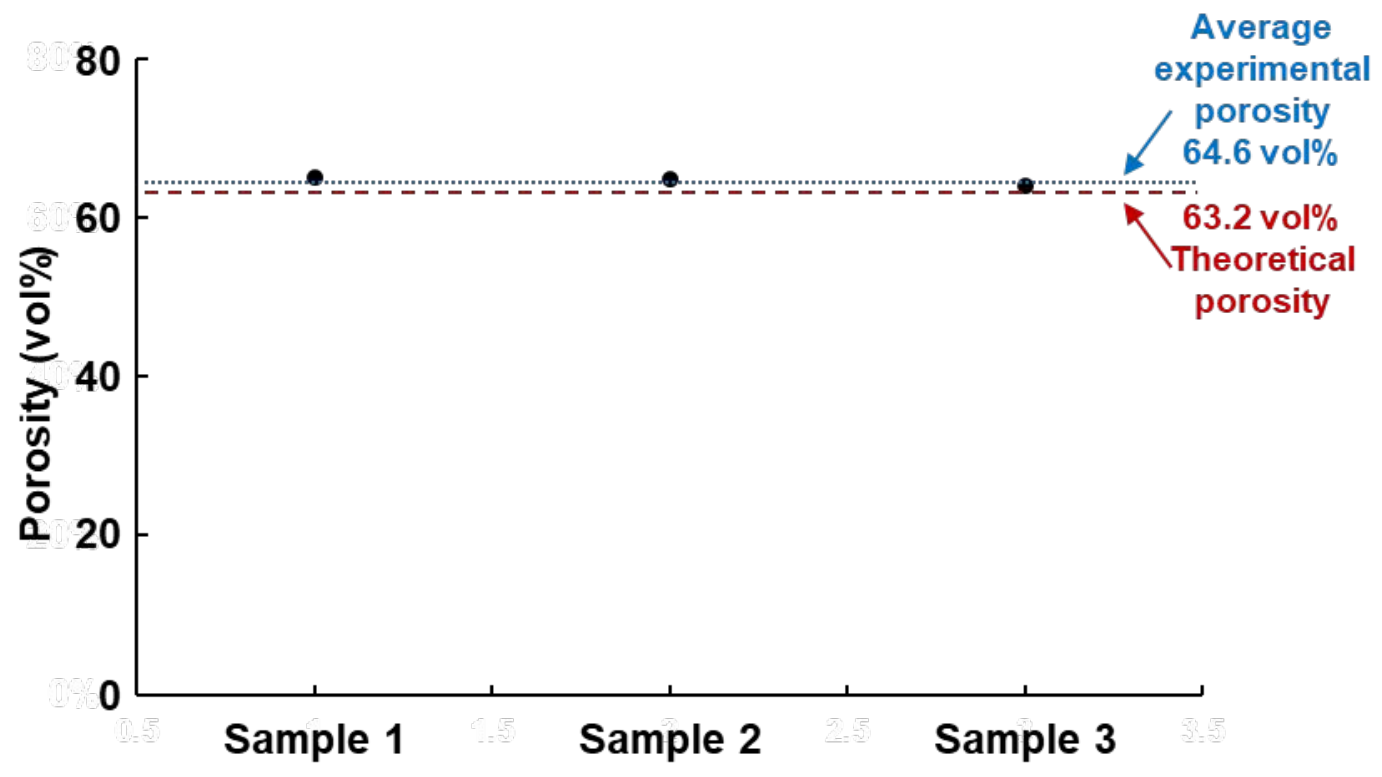

Figure S11. Plot of experimental and theoretical porosity of three samples with approximately 63 vol\% porosity prepared from ink composition listed in Table S3.

Table S4. Paraffin wax loading in paraffin/photopolymer composites and approximate porosity of material after extraction of paraffin.

\begin{tabular}{cccc}
\hline $\begin{array}{c}\text { Density of } \\
\text { paraffin wax }\end{array}$ & $\begin{array}{c}\text { Density of } \\
\text { resin }\end{array}$ & $\begin{array}{c}\text { Paraffin wax } \\
\text { loading in }\end{array}$ & $\begin{array}{c}\text { Porosity of } \\
\text { porous material }\end{array}$ \\
\hline
\end{tabular}




\begin{tabular}{cccc}
\hline$(\mathrm{g} / \mathrm{mL})$ & $(\mathrm{g} / \mathrm{mL})$ & composite $(w \mathrm{t} \%)$ & $(\mathrm{vol} \%)$ \\
& & 0 & 0 \\
& & 20 & 22 \\
& & 30 & 33 \\
0.904 & 1.02745 & 40 & 43 \\
& & 50 & 53 \\
& 60 & 63 \\
& 70 & 73 \\
\hline
\end{tabular}

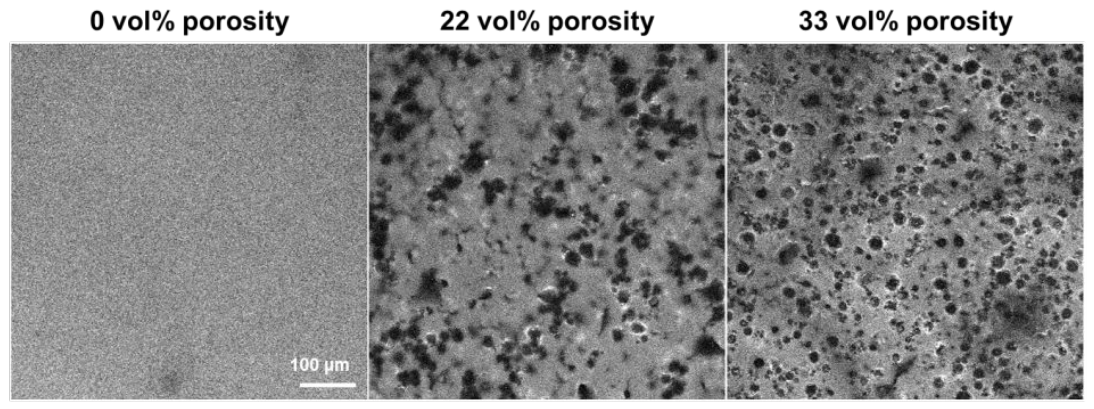

43 vol\% porosity

53 vol\% porosity

63 vol\% porosity

73 vol\% porosity

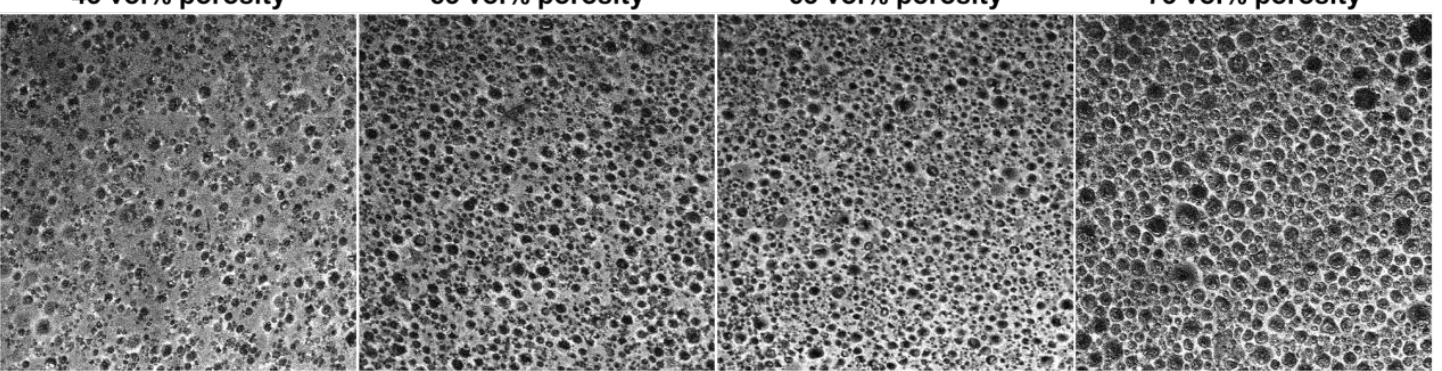

Figure S12. Confocal microscopy images of porous materials made from paraffin/photopolymer composite inks with varying wt $\%$ paraffin loading and thus varying vol\% pores. 


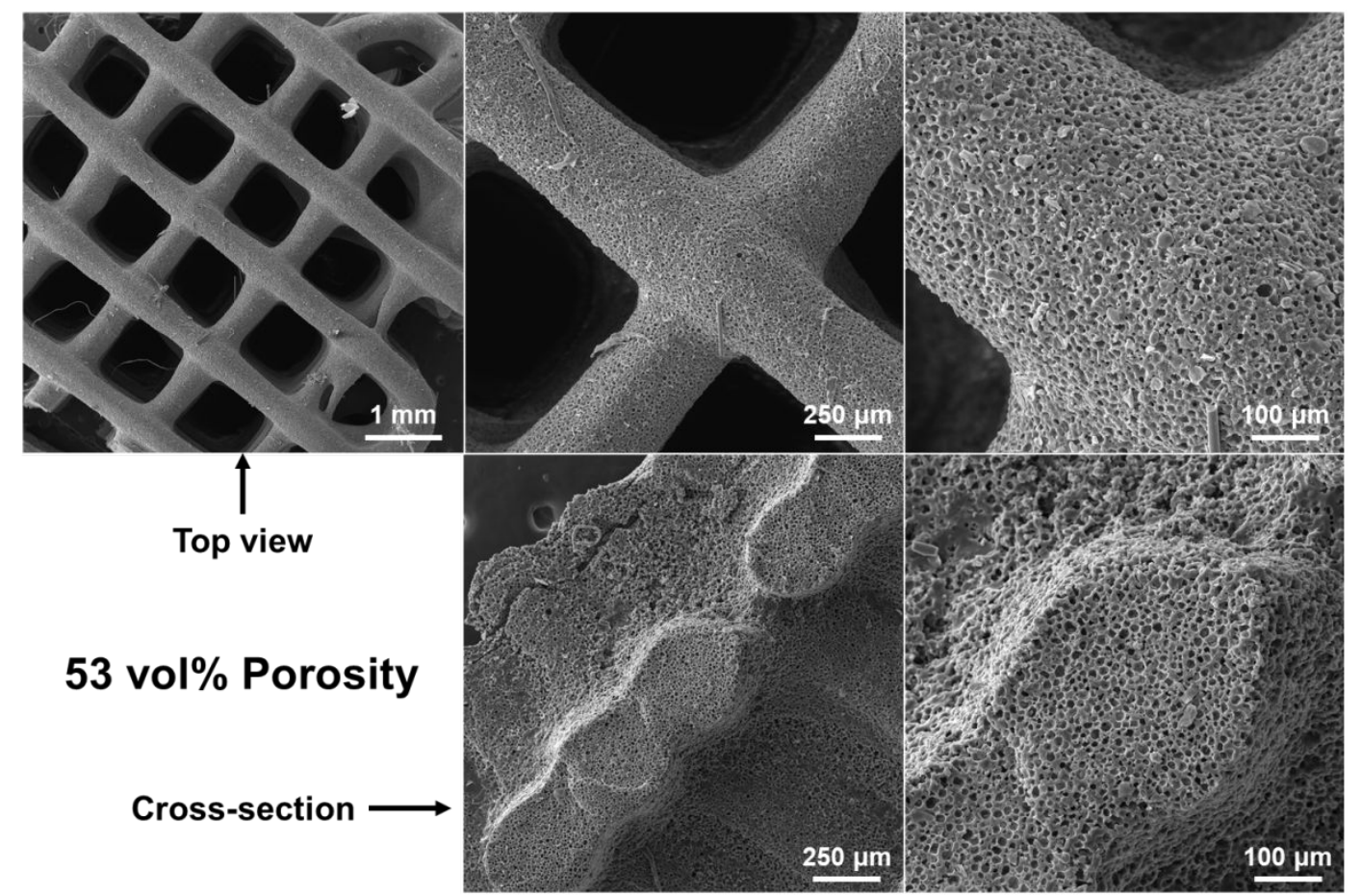

Figure S13. SEM images of 3D printed cubic lattice with 53 vol\% porosity.

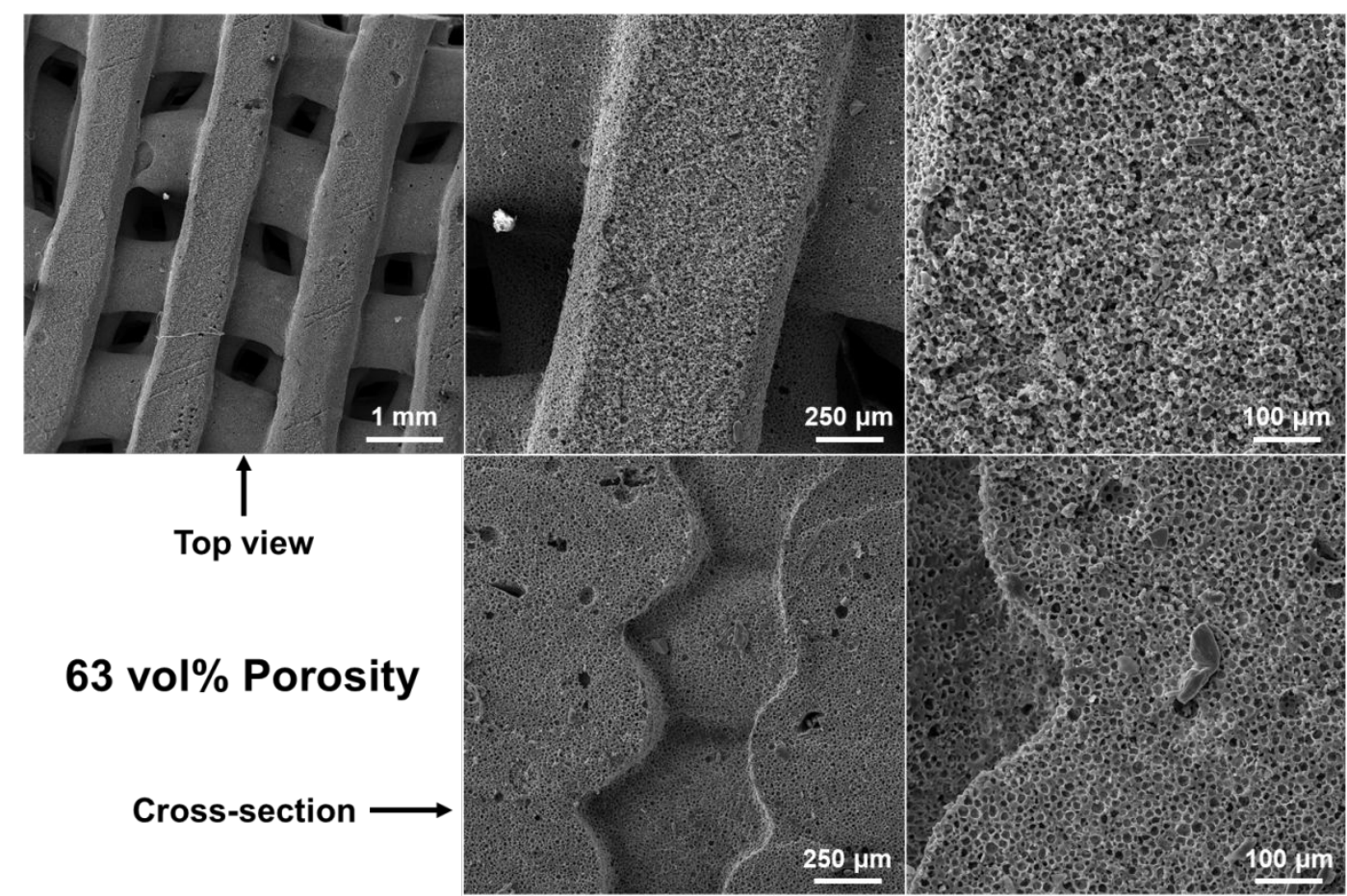

Figure S14. SEM images of 3D printed cubic lattice with 63 vol\% porosity. 


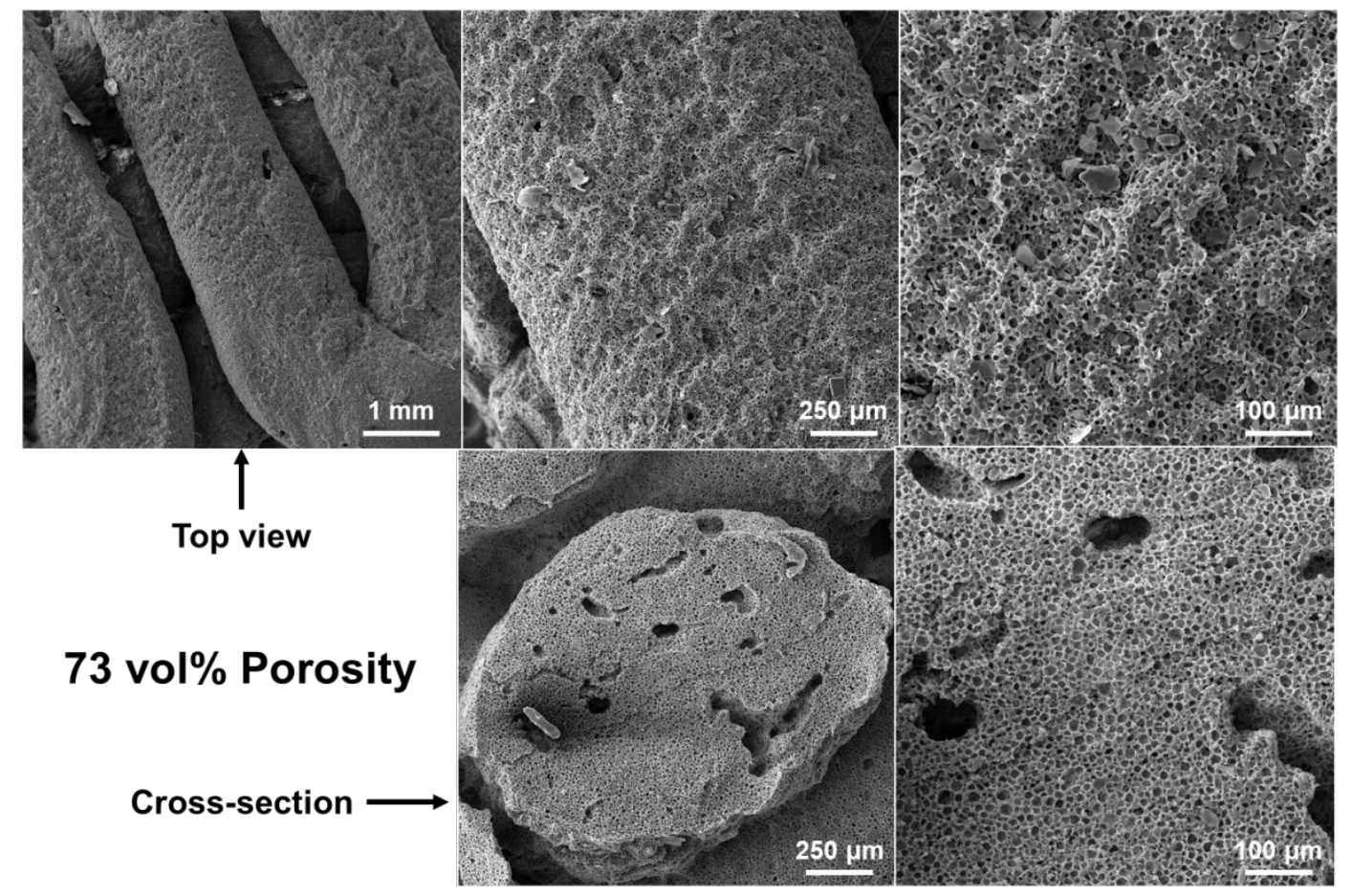

Figure S15. SEM images of 3D printed cubic lattice with 73 vol\% porosity.

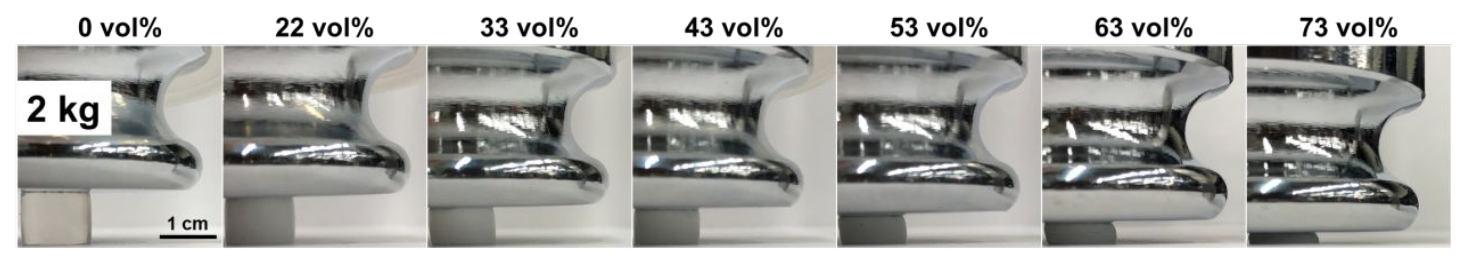

Figure S16. Digital images of cylinders of varying porosity levels compressed by a $2 \mathrm{~kg}$ weight. 


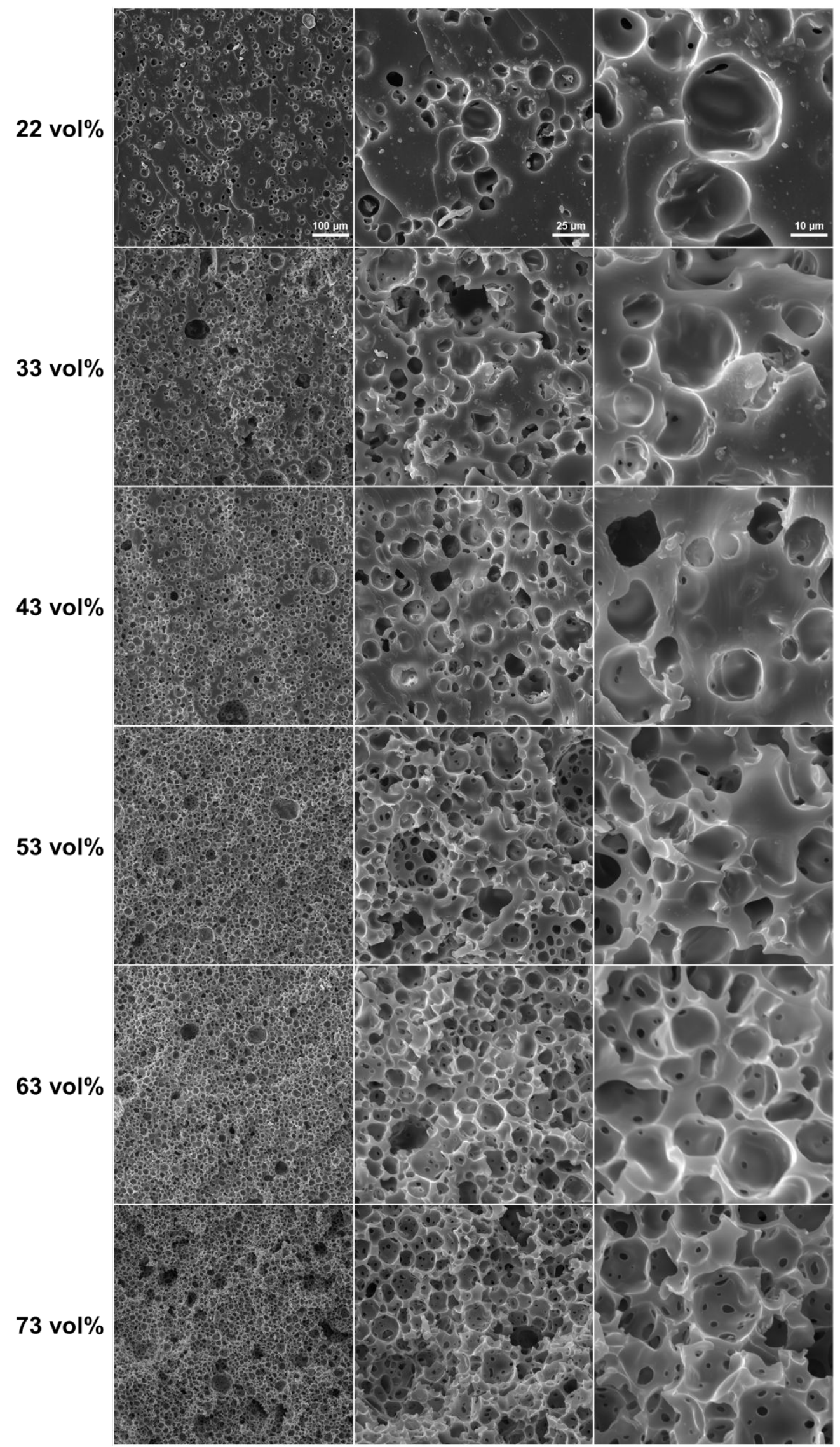

Figure S17. SEM images of fractured surfaces of tensile samples of varying levels of porosity. 


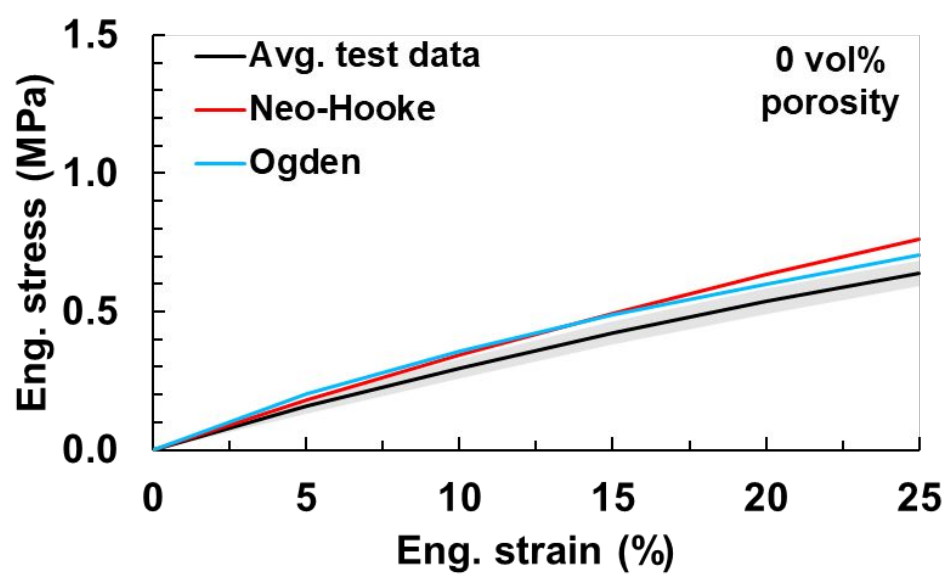

Figure S18. Stress-strain curves of the bulk resin with 0 vol\% porosity.

Table S5. Material constants used in the Neo-Hookean and Ogden models.

\begin{tabular}{cccccc}
\hline & $c_{1}$ & $\mu_{1}$ & $\alpha_{1}$ & $\mu_{2}$ & $\alpha_{2}$ \\
\hline Neo-Hooke & 0.5057 & - & - & - & - \\
Ogden & - & 0.7899 & 2.0000 & 0.2561 & -2.0000 \\
\hline
\end{tabular}

(A)

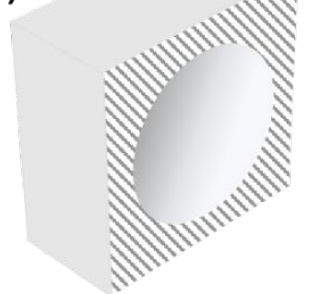

(D)

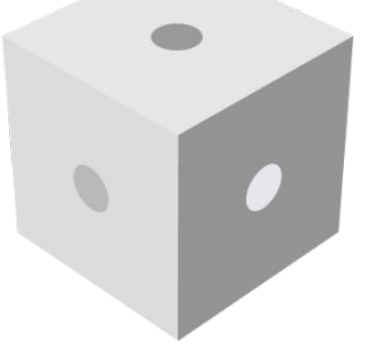

(B)

(E)
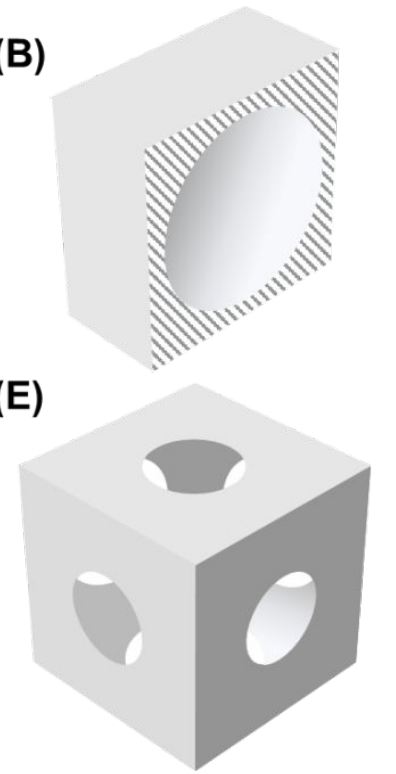

(C)

(F)
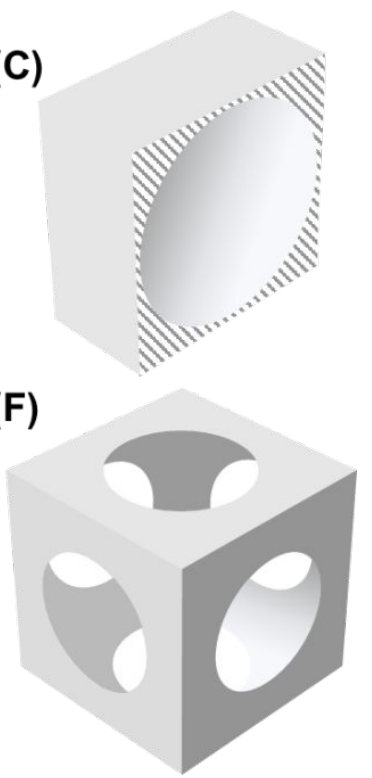

Figure S19. (A-C) Cross-sections of representative volume elements (RVEs) of porous materials with (A) 22 vol\%, (B) 33 vol\%, (C) 43 vol\% porosity; (D-F) full RVEs of porous materials with (D) 53 vol\%, (E) 63 vol\%, and (F) 73 vol\% porosity. 

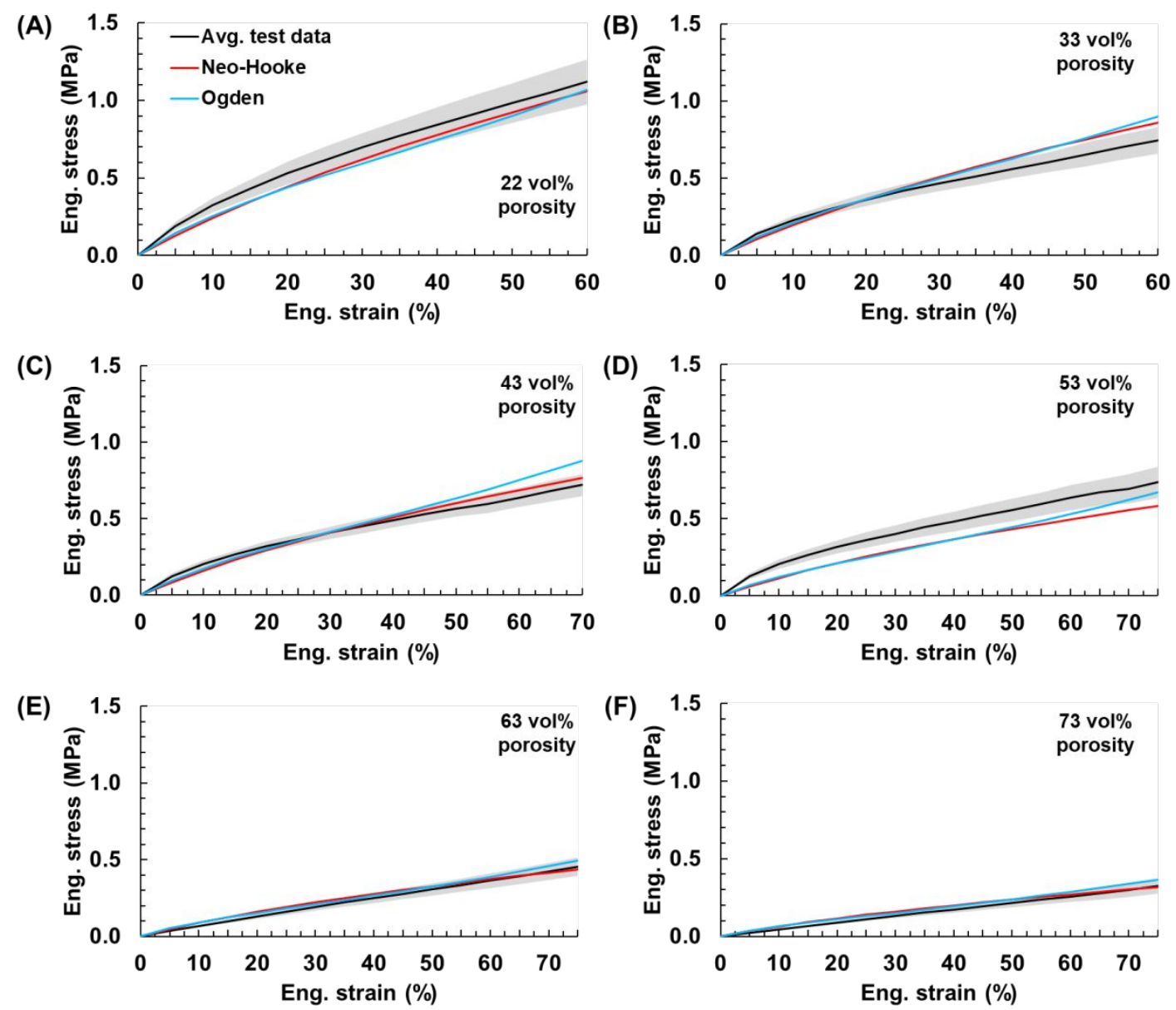

Figure S20. Stress-strain curves of porous materials with (A) $22 \mathrm{vol} \%$, (B) $33 \mathrm{vol} \%$, (C) 43 vol\%, (D) 53 vol\%, (E) 63 vol\%, and (F) 73 vol\% pores.

(A)

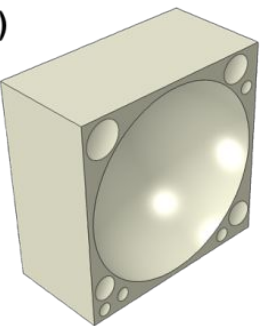

53 vol\% porosity Closed-cell foam mode
(B)

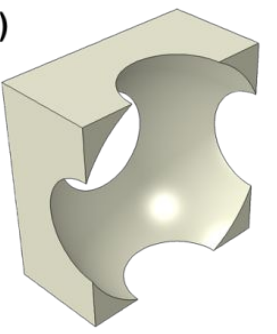

53 vol\% porosity Open-cell foam model

Figure S21. Possible cross-sections of RVEs of the 53 vol\% porosity sample as (A) a closed-cell foam and (B) an open-cell foam. 


\section{Supplementary Methods: Finite Element Analysis Methodology}

Strain Energy Function:

Hyperelastic materials can be described using a form of strain energy density of principal stretch, $\lambda$, or deformation invariants such as $I_{1}, I_{2}$, and $I_{3}$. Assuming that the $3 \mathrm{D}$ printed porous material in the present study was incompressible and isotropic, mechanical behavior was predicted by the Neo-Hookean and Ogden models. The strain energy density of a hyperelastic material is

$W=W\left[I_{1}(\boldsymbol{C}), I_{2}(\boldsymbol{C}), I_{3}(\boldsymbol{C})\right] \#(\mathrm{~s})$

where $C$ is the right Cauchy-Green deformation tensor, describing the deformation in the reference coordinate. The invariants of $C$ are

$I_{1}=\operatorname{tr}(\boldsymbol{C})=\lambda_{1}^{2}+\lambda_{2}^{2}+\lambda_{3}^{2} \#(\mathrm{~S})$

$I_{2}=\frac{1}{2}\left\{(\operatorname{tr}(\boldsymbol{C}))^{2}-\operatorname{tr}\left(\boldsymbol{C}^{2}\right)\right\}=\lambda_{1}^{2} \lambda_{2}^{2}+\lambda_{2}^{2} \lambda_{3}^{2}+\lambda_{3}^{2} \lambda_{1}^{2} \#(\mathrm{~S})$

$I_{3}=\operatorname{det}(\boldsymbol{C})=\lambda_{1}^{2} \lambda_{2}^{2} \lambda_{3}^{2} \#(\mathrm{~S})$

The strain energy density was constructed with principal stretches based on the molecular theory; the strain energy function was defined by the Neo-Hookean form as follows: ${ }^{22}$

$W=\frac{1}{2} \mu\left(\lambda_{1}^{2}+\lambda_{2}^{2}+\lambda_{3}^{2}-3\right) \#(\mathrm{~S})$

where $\mu$ is the shear modulus. For uniaxial tension, the strain energy density and nominal stress are expressed with the uniaxial stretch ratio and given by

$W\left(\lambda_{1}\right)=c_{1}\left(\lambda_{1}^{2}+2 \lambda_{1}^{-1}-3\right) \#(\mathrm{~S})$

Accordingly, the nominal stress of the Neo-Hookean model is expressed by

$\sigma_{1}=2 c_{1}\left(\lambda_{1}-\lambda_{1}^{-2}\right) \#(\mathrm{~S})$

The strain energy density in terms of a linear combination of principal stretches was introduced by Ogden. ${ }^{23}$ The strain energy density of the Ogden model is

$W\left(\lambda_{1}, \lambda_{2}, \lambda_{3}\right)=\sum_{i=1}^{m} \frac{\mu_{i}}{\alpha_{i}}\left(\lambda_{1}^{\alpha_{i}}+\lambda_{2}^{\alpha_{i}}+\lambda_{3}^{\alpha_{i}}-3\right) \#(\mathrm{~S})$

where $\mu_{i}$ and $\alpha_{i}$ are material constants describing shear behavior. For uniaxial tension, the strain energy density and nominal stress are

$W\left(\lambda_{1}\right)=\sum_{i=1}^{m} \frac{\mu_{i}}{\alpha_{i}}\left(\lambda_{1}^{\alpha_{i}}+2 \lambda_{1}^{-\frac{1}{2} \alpha_{i}}-3\right) \#(\mathrm{~S})$

$\sigma_{1}=\sum_{i=1}^{m} \mu_{i}\left(\lambda_{1}^{\alpha_{i}-1}-\lambda_{1}^{-\frac{1}{2} \alpha_{i}-1}\right) \#(\mathrm{~S})$

\section{Periodic Boundary Conditions:}

To simulate the experimental tensile tests, the bulk porous materials resulting from the removal of the paraffin wax were considered as a set of periodically arranged RVEs comprising the structure of an ASTM D412 Type C tensile sample. The deformation of each opposite side of the RVE should be the same to satisfy the periodic boundary conditions, which are provided in the SI. This implies that the deformation of each RVE in the porous material is identical and that the boundary conditions of neighboring RVEs are the same.

$$
\begin{aligned}
& u_{O C C^{\prime} O^{\prime}}-u_{A B B^{\prime} A^{\prime}}+\hat{u}=0 \\
& v_{O A A^{\prime} O^{\prime}}-v_{C B B^{\prime} C^{\prime}}+\hat{v}=0
\end{aligned}
$$




$$
\begin{gathered}
w_{O A B C}-w_{O^{\prime} A^{\prime} B^{\prime} C^{\prime}}+\hat{w}=0 \\
u_{O C C^{\prime} O^{\prime}}=v_{O A A^{\prime} O^{\prime}}=w_{O A B C}=0 \#(\mathrm{~S} 11 .) \\
\hat{u}=u_{A^{\prime} B^{\prime}} \\
\hat{v}=v_{B^{\prime} C^{\prime}} \\
\hat{w}=\text { specified }
\end{gathered}
$$

where $u, v$, and $w$ are the displacements of the $x, y$, and $z$ axes, respectively, and $O$, $O^{\prime}, A, A^{\prime}, B, B^{\prime}, C$, and $C^{\prime}$ are points used to define the faces and axes of an RVE as shown in Figure S22. $\hat{u}, \hat{v}$, and $\hat{w}$ are prescribed values.

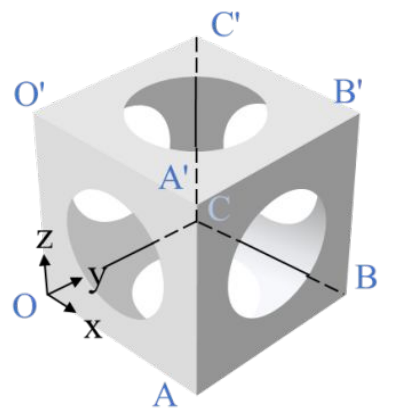

Figure S22. A representative RVE used in this study. Each RVE was modeled with unit length and a spherical void in the center. 